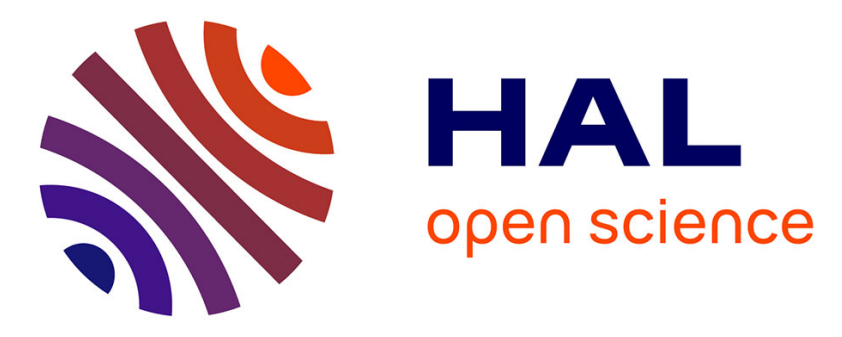

\title{
Concept and architecture of a new apparatus for cylindrical form measurement with a nanometric level of accuracy
}

Alain Vissiere, Hichem Nouira, Mohamed Damak, Olivier Gibaru, Jean Marie

David

\section{To cite this version:}

Alain Vissiere, Hichem Nouira, Mohamed Damak, Olivier Gibaru, Jean Marie David. Concept and architecture of a new apparatus for cylindrical form measurement with a nanometric level of accuracy. Measurement Science and Technology, 2012, 23 (9), pp.10. 10.1088/0957-0233/23/9/094014 . hal01084368

\section{HAL Id: hal-01084368 \\ https://hal.science/hal-01084368}

Submitted on 20 Nov 2014

HAL is a multi-disciplinary open access archive for the deposit and dissemination of scientific research documents, whether they are published or not. The documents may come from teaching and research institutions in France or abroad, or from public or private research centers.
L'archive ouverte pluridisciplinaire $\mathbf{H A L}$, est destinée au dépôt et à la diffusion de documents scientifiques de niveau recherche, publiés ou non, émanant des établissements d'enseignement et de recherche français ou étrangers, des laboratoires publics ou privés. 


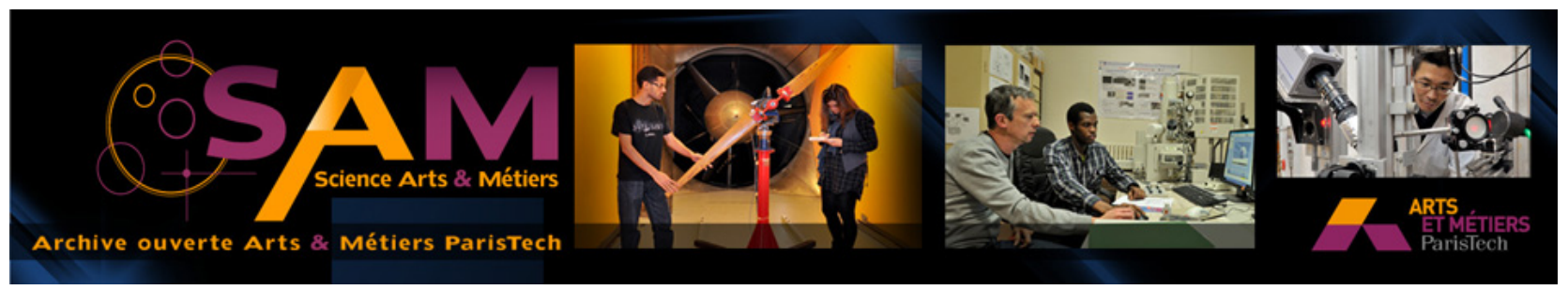

\section{Science Arts \& Métiers (SAM)}

is an open access repository that collects the work of Arts et Métiers ParisTech researchers and makes it freely available over the web where possible.

This is an author-deposited version published in: http://sam.ensam.eu

Handle ID: .http://hdl.handle.net/10985/8944

\section{To cite this version :}

Alain VISSIERE, Hichem NOUIRA, Mohamed DAMAK, Jean Marie DAVID, Olivier GIBARU Concept and architecture of a new apparatus for cylindrical form measurement with a nanometric level of accuracy - Measurement Science and Technology - Vol. 23, n9, p.10 - 2012 


\title{
Concept and architecture of a new apparatus for cylindrical form measurement with a nanometric level of accuracy
}

\author{
A Vissiere ${ }^{1,2}$, H Nouira $^{3,4}$, M Damak $^{1,2}$, O Gibaru $^{1}$ and J-M David $^{1}$ \\ ${ }^{1}$ Ecole Nationale Supérieure d'Arts and Métiers de Lille (Arts et Métiers ParisTech), (LSIS), \\ 8 Boulevard Louis XIV, 59046 Lille, France \\ ${ }^{2}$ GEOMNIA: Advanced 3D Engineering and Software Solutions, 165 Avenue de Bretagne, \\ Eura Technologies 59000 Lille, France \\ ${ }^{3}$ Laboratoire National de Métrologie et d'Essais (LNE), Dimensional Metrology Department, \\ 1 Rue Gaston Boissier, 75015 Paris, France \\ E-mail: hichem.nouira@lne.fr
}

\begin{abstract}
In relation to the industrial need and to the progress of technology, Laboratoire National de Métrologie et d'Essais (LNE) would like to improve the measurement of its primary pressure standards, spherical and flick standards. The spherical and flick standards are, respectively, used to calibrate the spindle motion error and the probe, which equip commercial conventional cylindricity-measuring machines. The primary pressure standards are obtained using pressure balances equipped with rotary pistons. To reach a relative uncertainty of $10^{-6}$ in the pressure measurement, it is necessary to know the diameters of both the piston and the cylinder with an uncertainty of $5 \mathrm{~nm}$ for a piston diameter of $10 \mathrm{~mm}$. Conventional machines are not able to reach such an uncertainty level. That is why the development of a new machine is necessary. The purpose of this paper is to present the concepts and the architecture adopted in the development of the new equipment dedicated to cylindricity measurement at a nanometric level of a accuracy. The choice of these concepts is based on the analysis of the uncertainty sources encountered in conventional architectures. The architecture of the new ultra-high equipment as well as the associated calibration procedures will be described and detailed.
\end{abstract}

Keywords: dimensional metrology, precision engineering, form measurement, uncertainty, form error

(Some figures may appear in colour only in the online journal)

\section{Introduction}

This work is part of a project whose objective is to develop a new ultra-high precision cylindrical measurement machine. The equipment is mainly dedicated to measure standards, such as flick standards and spheres, which are used for the calibration of conventional cylindricity-measuring machines, with an uncertainty level of a few nanometers.

\footnotetext{
${ }^{4}$ Author to whom any correspondence should be addressed
}

The project also has the objective of improving the design and use of nanometric pressure balances equipped with rotary pistons. These pressure balances are used as primary pressure standards and integrated in balances intended for the calibration of significant masses. They are based on a piston/cylinder set of very high geometrical quality typically achieved via a lapping process of very hard materials. Tightness between both parts is achieved through a very small clearance (of around $1 \mu \mathrm{m}$ ), which leaves space for laminar flow between the two surfaces as shown in figures 1 and 2. No 

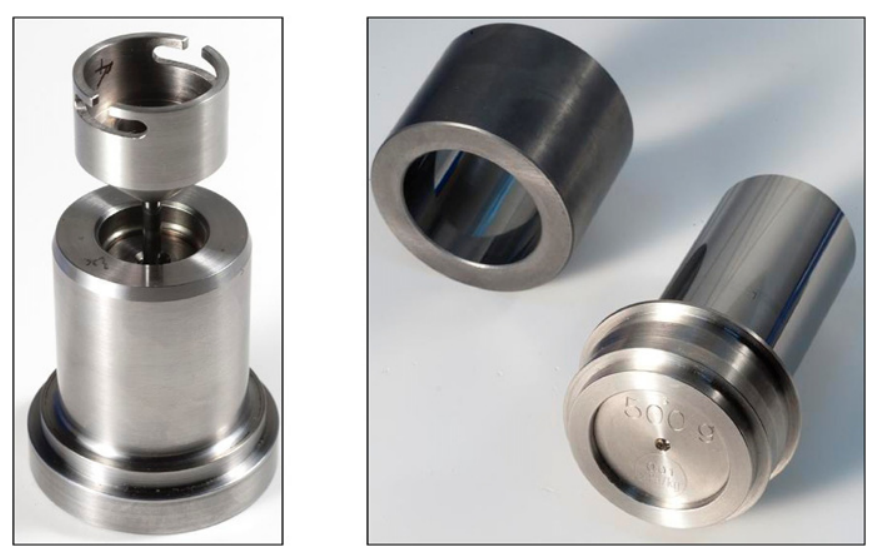

Figure 1. Piston and cylinder of a pressure standard piston-cylinder assembly.

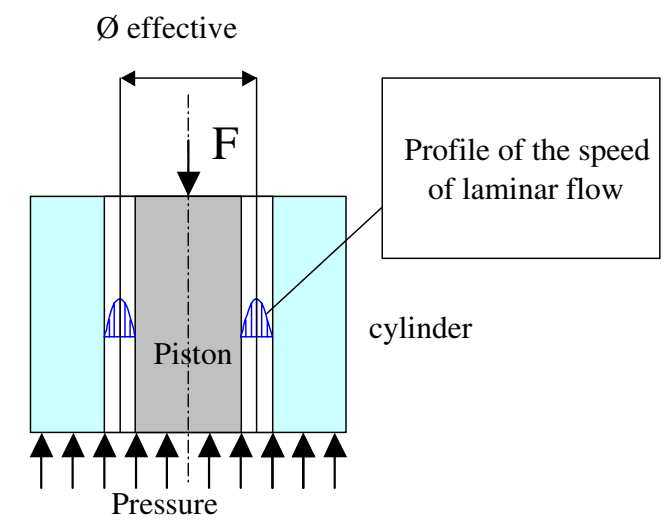

Figure 2. Pressure balance equipped with rotary piston.

joints with contacts that may create friction forces are used. A permanent rotating movement is maintained around the axis common to the two parts. This movement can prevent friction effects caused by the contact between two solids. The form of the cylinder is optimized so that the flow can recenter the piston and prevent it from sticking.

The establishment of pressure references with a relative uncertainty of $10^{-6}$ gives rise to the need to know the diameter of pistons and cylinders with uncertainties of approximately $0.5 \times 10^{-6}$. That leads to having an uncertainty of $5 \mathrm{~nm}$ for a piston with a diameter of $10 \mathrm{~mm}$. With the form measurement of cylinders and pistons, we will be able to apply flow patterns to the piston/cylinder clearance. Laminar flow pattern modeling gives results that are consistent with reality. We may be able to link the form flaws of cylinders and pistons with their influence on the stresses exerted on the piston.

To achieve the targeted level of uncertainty, new equipment under development applies the dissociated metrology technique (DMT) well defined in $[1,2]$. The technique has already been tested in several achievements of LNE: ultra-high-precision angular machine [3], nanometric compound table [4] and AFM machine [5]. Methods for eliminating errors will be applied to calibrate the machine. Finally, some technology solutions will be developed to control the thermal behavior of the equipment.
With this equipment, we aim for an uncertainty less than $5 \mathrm{~nm}$ for the roundness measurement and an uncertainty less than $10 \mathrm{~nm}$ for straightness and cylindricity assessments in the entire measurement volume (standards with a maximum diameter of $100 \mathrm{~mm}$ and a height of $150 \mathrm{~mm}$ ). The target uncertainty represents a challenge compared to the best uncertainties reported in the BIPM's Key Comparison Database (KCDB) [6] and mainly achieved by the German metrologists of PTB (National Metrology Institute of Germany). These uncertainties are as follows:

- $50 \mathrm{~nm}$ for the cylindricity assessment of a flick standard,

- $50 \mathrm{~nm}$ for the straightness assessment of a flick standard,

- $5 \mathrm{~nm}$ for roundness assessment of sphere and cylinder standards.

In this paper, the analysis of the error, which causes inherent inaccuracy in conventional and industrial machines for cylindricity measurement, as well as the limits of error corrections on these machines, will be evoked. The evolution from a conventional architecture toward an architecture integrating the DMT technique will be presented and discussed.

\section{The analysis of conventional equipment for cylindricity measurement}

On conventional machines, measurement is performed through the comparison of the part's form with a high-quality movement. The roundness of a part is measured by subjecting it to a high-quality rotation movement and monitoring its surface with a fixed probe. The measurement of more complex characteristics, like cylindricity, requires combined movements, which are performed by a more universal machine for metrology. Machines typically have a 'series' loop structure. Series structures are made up of a succession of solids joined by customizable linkages that are able to generate relative positions or movements between two solids. This type of architecture can be found on most coordinate-measuring machines.

\subsection{The analysis of the metrology loop}

Generally, a concept called 'metrology loop' is used to identify the causes of errors. A metrology loop can be defined as a conceptual line going through all solids, sensors and joints of a machine and determining the relative position of the probe with respect to the artifact. Figure 3 shows an example of conventional and industrial geometry measurement machine. Figure 4 describes the kinematic scheme of this type of machine and gives an idea about the metrology loop that reflects its metrological performance.

In the case of cylindrical artifacts, the surface to be measured is scanned using three serial linkages that represent the following essential components of the metrology loop.

- A revolute joint between the precision spindle (1) and the supporting structure (2). 


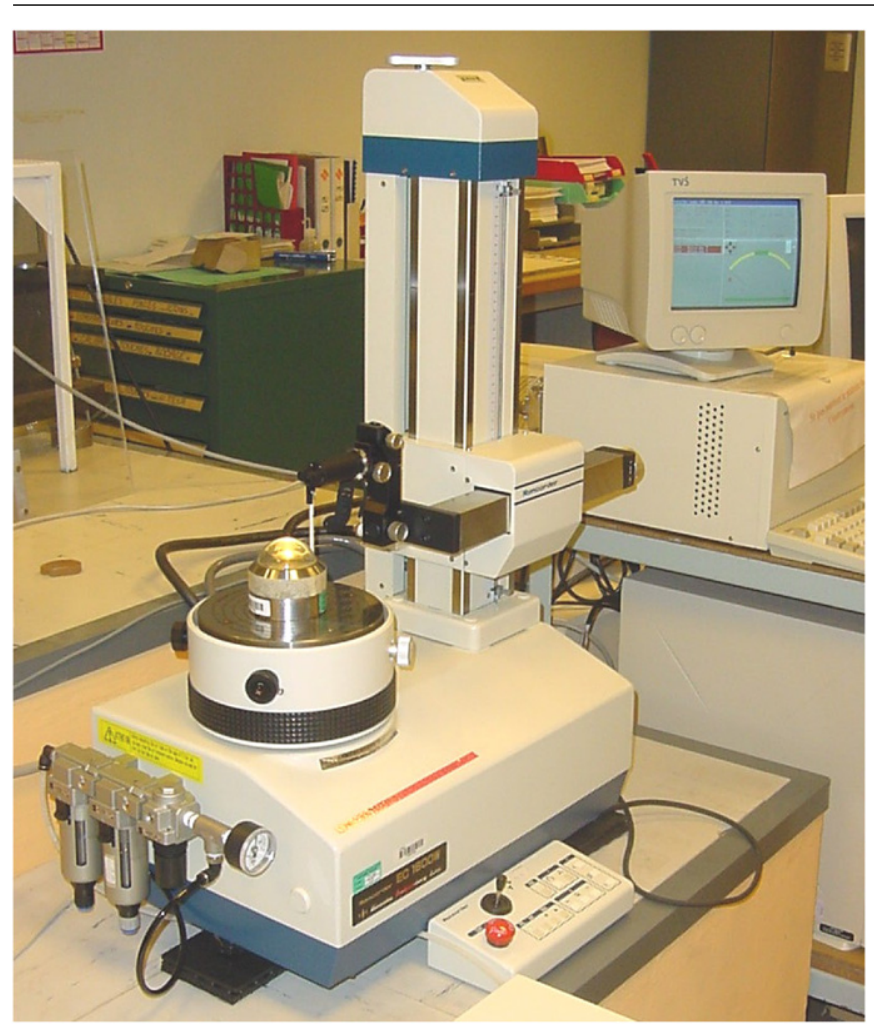

Figure 3. Example of a conventional geometry measurement machine: the KOSAKA machine.

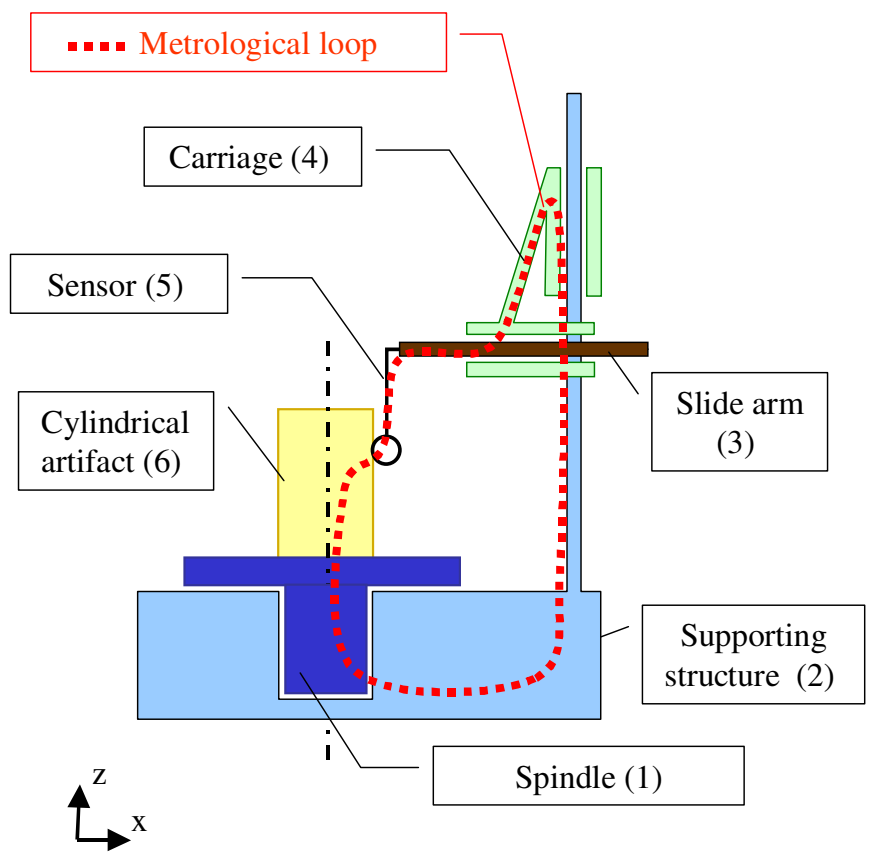

Figure 4. Kinetic diagram of a conventional machine and identification of the metrology loop.

- A bi-dimensional scan is performed on a radial plane using two serial guide linkages. The first mechanical guiding element is between column (2) and carriage (4) and allows scanning along the vertical axis. The second mechanical guiding element is between carriage (4) and arm (3) and allows scanning along the horizontal axis.
An encoder and rulers are used to determine the coordinates along the scanning axes. The measuring probe (5), in contact with the artifact (6) to be measured, represents the last component of the metrology loop.

\subsection{Sources of disturbance}

Any non-controlled dimensional change has an impact on the uncertainty. Dimensional changes of the metrological loop can be divided mainly into two categories: corrigible and incorrigible errors.

Identifiable changes are mainly caused by the following.

- Errors in metrological reference standards like encoders and rulers.

- Movement errors caused by the form of the surface of the guiding systems.

- Errors of relative position between movements.

- Errors caused by elastic deformation of components and mechanical guiding systems under the effect of stresses that are perfectly controlled and repeatable.

\subsection{Corrigible errors}

As presented in figure 3 , the horizontal arm deflects according to the horizontal position (along the $x$-axis) of the probe. However, the deflection is higher when the position of the probe is as far as possible from the column, and inversely. This causes the probe to go down. When the arm is vertically positioned as high as possible, the deflection of the column is also larger and inversely. To reduce this error source, usually, the constructors design mechanical guiding systems whose shape would correct these deformation effects [4]. However, the effect of this method is limited: the modification of a guiding system is based on one variable, whereas for the machine given as an example, the correction shall be based on two variables: $x$ and $z$.

There are some achievements that implemented mechanical systems for subjecting the machine to stresses. The flexibility of corrections made through computers removes this difficulty, provided that the content of the calibrations to perform is clearly defined. Repeating calibrations leads to an improvement of the quality of the corrections [2]. Of course, this correction possibility only applies to errors that are repeatable and describable.

\subsection{Incorrigible errors}

Incorrigible modifications of the metrology loop are caused by the following.

- Non-repeatable errors of mechanical guidance systems.

- Differential thermal expansions.

- The effect of vibrations.

- Gaps and effect of friction.

These errors cannot be corrected using the method mentioned previously. 


\section{The principle of dissociated metrological structure}

\subsection{DMT principle}

The supporting structure of an industrial machine can be defined as an assembly of components that maintain the relative position of the probe with respect to the target. The DMT principle consists in dissociating the metrology loop from the 'load-bearing loop' [1-4]. This allows components of the metrology loop to be used only for the communication of position information. The metrology loop is relieved of any other function, in particular the support function, and can be optimized.

This makes the following possible.

- Avoid deformations in the metrology loop caused by nonpredictable loads. The metrological structure will only have to bear its own weight.

- Create linkages between the components of the metrology loop using probes. These so-called metrological linkages can communicate position information in a much more repeatable way than any mechanical linkage. Metrological linkages are not sensitive to loads. The redundancy of the information obtained through an increase in the number of sensors improves the quality and the reliability of measurements and is even a permanent means of autoverification.

- Reduce the effect of thermal disturbances and drifts.

\subsection{Calibration of the machine}

The following two complementary corrections of coordinates must be performed when handling the calibration of the machine.

- The first correction consists in interpreting the data from metrological linkages leading to a correction 'at the tip of the probe'. At this stage, the machine becomes a machine whose mechanical guiding systems have perfect repeatability.

- The references of the machine's movements have geometrical errors that result in machine errors. The machine is calibrated by measuring standards and by applying error separation methods like reversal, multistep and multi-probe methods. The acceptable range of the geometrical error of the references is only limited by the travel range of the probes used.

\subsection{Outcome}

The implementation of DMT in a machine leads to the following.

- Permanent verification of the machine's precision quality.

- The ability to maintain an ultra-high precision level for a large dimension structure.

- The ability to make machines less sensitive to environmental disturbances (thermal variations, vibrations).

- Great savings in the making of the machine: there is no need to use ultra-high-precision guidance elements.

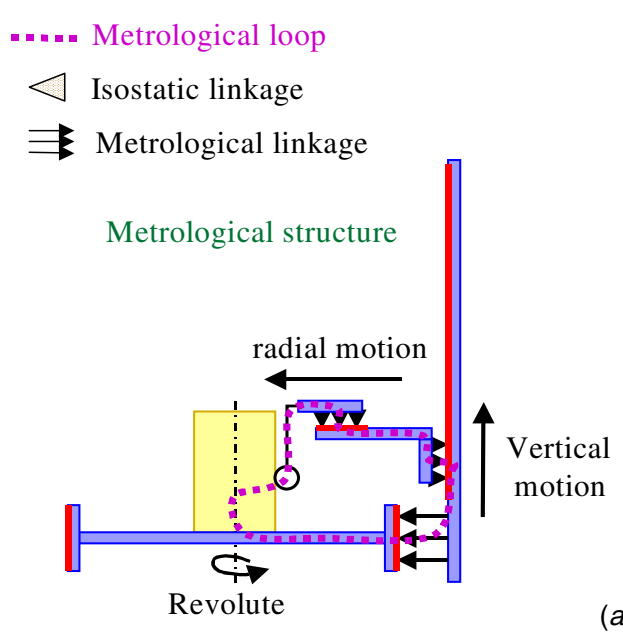

(a)

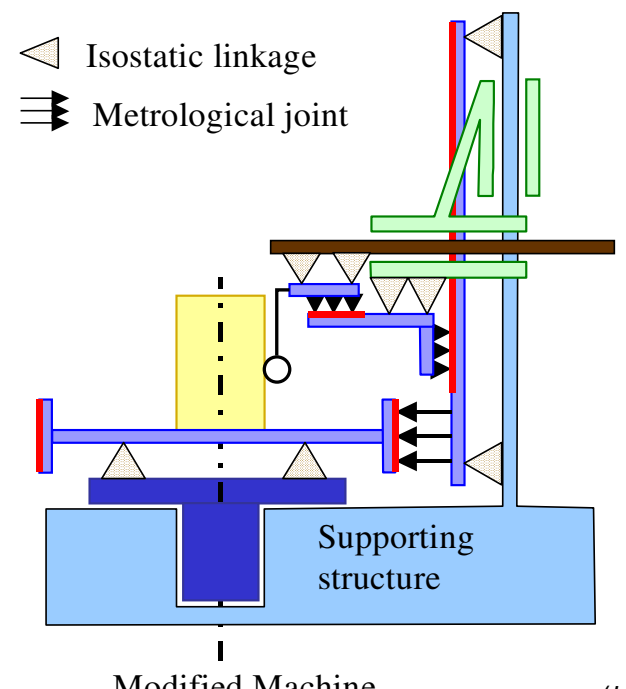

(b)

Figure 5. Conventional machine: application of the principle of dissociated metrological structure (DMT).

\section{The application of the DMT principle to a conventional form measurement machine}

\subsection{Description of the architecture}

The architecture presented in figure 5 is obtained by applying the principle of dissociated metrological structure to a conventional cylindricity-measuring machine. The metrology loop and the supporting loop are separated. Linkages between corresponding elements of the two structures are isostatic linkages that allow deformation to be prevented in the components of the metrology loop. Parts of these principles are applied in machine MFU8 and MFU110WP of brand MAHR modified by PTB [7].

\subsection{Optimizing the application of the principle of dissociated metrological structure}

The application of the DMT principle can be optimized by reducing the number of movements. Then, the proposed scheme presented in figure 6 can be considered and the 


\section{" - " Metrological loop \\ $\rightarrow$ Sensor \\ Metrlogical structure}

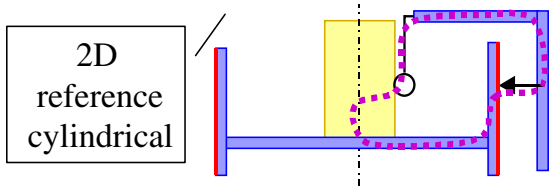

Figure 6. Optimized metrological structure.

$$
\begin{aligned}
& \longrightarrow \text {. Metrological loop } \\
& \rightarrow \text { Sensor } \\
& \text { Metrological structure }
\end{aligned}
$$

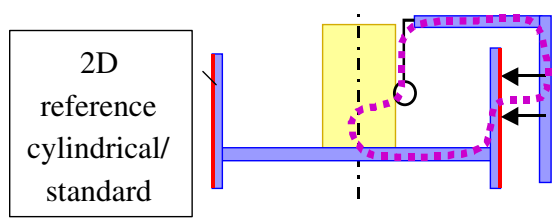

Figure 7. Use of two sensors to reproduce Abbe's principle.

precision reference cylinder can be a cylindrical sleeve with a two-dimensional reference surface.

The architecture of the machine contains only one reference probe, which is aligned with the probe that measures the part with perfect respect of Abbe's principle [8]. This architecture is close to the one used on the diameter and form measurement machine made by Neugebauer et al in PTB [9].

In the diagram presented in figure 7 , the number of probes focused on the reference cylinder has been doubled and the reconstituted Abbe's principle is respected. Then, Abbe's principle can be verified accurately, and the use of two reference probes instead of one divides the uncertainty by a factor of 1.4. The performed architecture of the machine operates in a much more elementary way. Two surfaces can be easily compared using point by point correspondence. Therefore, it is no longer necessary to differentiate straightness error from roundness error. This is called 'global cylindricity uncertainty'.

The offset of the probe from the generator, which can be caused by both the eccentricity of the reference cylinder and the artifact, as well as the spindle motion error, of the cylindrical reference and/or artifact, influences the accuracy of the roundness assessment (see figure 8). As a consequence, to complete measurement of roundness on artifacts with a small diameter $R(R \leqslant 5 \mathrm{~mm})$ with a nanometric level of accuracy, the offset error $\delta$ should be absolutely considered and compensated. For example, for a part of $2 \mathrm{~mm}$ of diameter, an offset of $5 \mu \mathrm{m}$ generates an error of $12 \mathrm{~nm}$. This value does not comply with the targeted uncertainties. However, the following two solutions are proposed in order to minimize the probe offset error.

- Implementing mechanical guiding elements with ultrahigh quality of motion: air bearing spindle and air bearing linear stage [9].

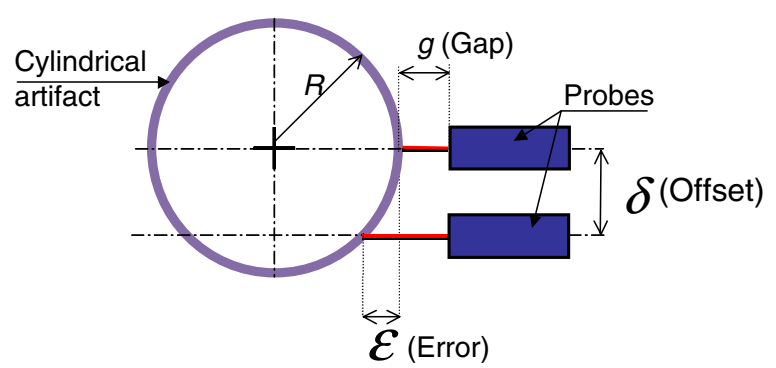

Figure 8. Description of the probe offset error caused by the lateral motion of the artifact.

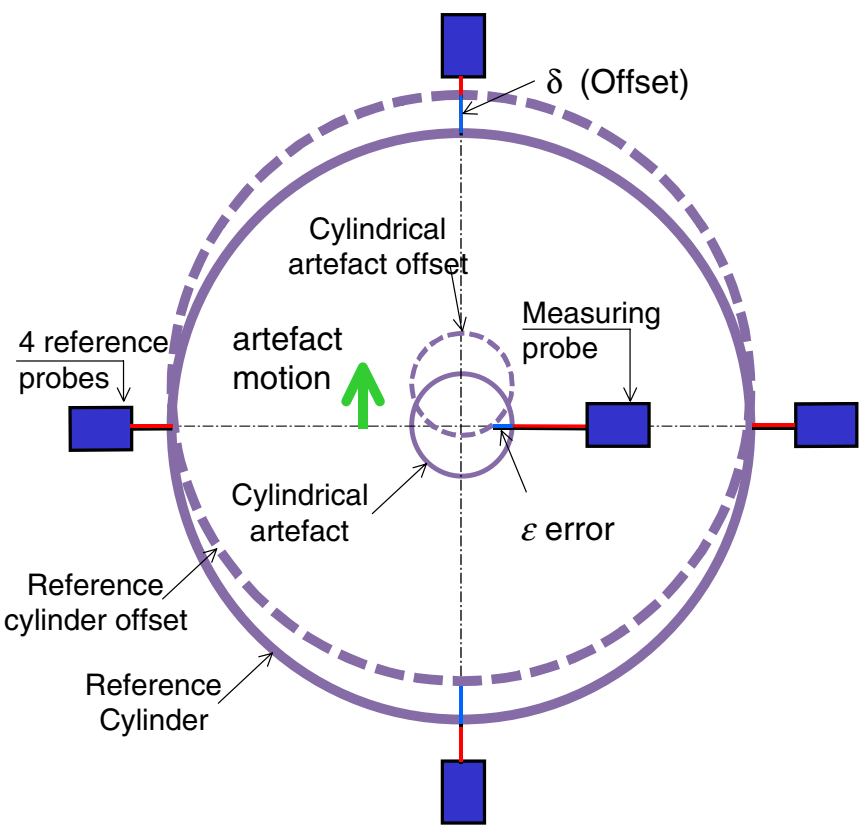

Figure 9. Description of the compensation of the probe offset error.

- Combining both the classical mechanical guiding system and the centering system in real time.

The second solution was adopted for the new ultra-high precision cylindricity machine, which will be equipped by a ball bearing with a maximum concentricity of $\pm 5 \mu \mathrm{m}$ and a centering system able to detect the probe offset values. The measurement of the offset will be realized thanks to two probes located at an angle of $90^{\circ}$ with respect to the first probe as described in figure 9. Then, knowledge of the value of the probe offset $\delta$ allows compensation of the $\varepsilon$ error in real time, by using a special mechanical system (compound tables $X$ and $Y$ ), which ensures a motion step with nanometer level accuracy, or by using the mathematical formulation as presented in equation (1) and demonstrated in [10]:

$$
\varepsilon=\frac{\delta^{2}}{2 R} \text {. }
$$

\subsection{Calibration of the reference cylinder: roundness and straightness}

The calibration of the proposed machine is based on the application of the modified multi-step form error separation technique. The conventional error separation method is 
detailed in [11, 12] and allows separation between the part form error and the synchronous motion error of the mechanical guiding systems (linear guiding system and aerostatic spindle).

The new modified multi-step method allows separation between the form errors of both the reference cylinder and the cylindrical artifact. The measurements are then never influenced by the quality of motion of the mechanical guidance elements. The modified multi-step method is applied with many height values by performing $N$ angular-spacing steps between both surfaces. The signal corresponding to the difference between the dataset obtained by both measuring and reference probes gives the deviation between the two functions of roundness forms of both surfaces. By calculating the average of the deviation functions, it is possible to obtain an imaginary form surface very close to the real form of the reference cylinder.

The weak point of this method is that an error with a periodicity of $N$ periods per turn cannot be detected. This drawback could be avoided by performing the measurement twice: once with $N$ angular-spacing steps and a second time with $P$ angular-spacing steps, with $N$ and $P$ being two coprime integers. If the relative position between both surfaces remains rigorously stationary during the time needed to scan the surfaces entirely, the roundness values obtained are indexed to each other and a cylindricity measurement can be obtained $[13,14]$.

The modified multi-step error separation method increases the duration of measurements significantly, but it also increases the number of measurements, leading to the decrease of measurement uncertainties.

The modified multi-step method can be applied using an automated angular indexing table under the control of two optical encoders. The table will ensure a perfectly stationary link between the two surfaces that are compared. It is not required to maintain perfect coaxiality between the two surfaces, but there should not be any relative movement on the nanometer level during the scanning process of both surfaces. The angular indexing table is made so as to comply with this requirement.

The evaluation of the straightness of the generators of the cylindrical surface is also considered. For this reason, the modified reversal method will be applied and detailed in $[13,14]$. The angular-spacing permutation is obtained by shifting the cylindrical artifact and the reference cylinder by half a turn. In order to avoid turning the probe, which could affect the calibration, an additional measuring probe opposite to the first probe is implemented as shown in figure 10 [14]. Similarly, the two opposite probes can be used to identify information concerning the conical form of the reference cylinder.

\subsection{Selecting reference probes (probes focused on the reference cylinder)}

On the machine, only contactless probes have to be used for sensing the reference cylinder. After an experimental testing and characterization of capacitance sensors with different electrode diameters (nonlinear residual, impact of the form

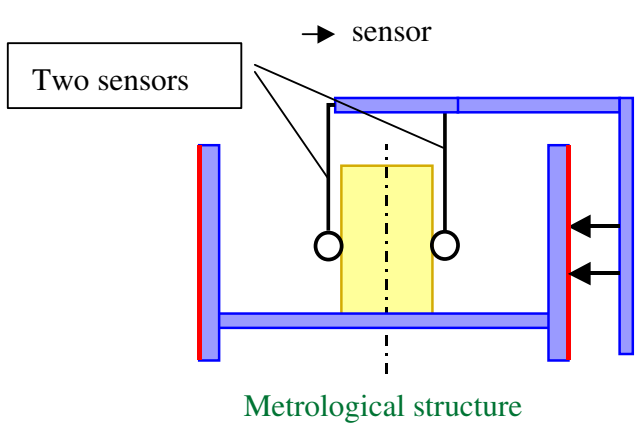

Figure 10. Calibration of taper and straightness.

of the target, slope, radial offset tests and stability) [10, 15, 16], the capacitance sensor whose electrode has a diameter of $5.5 \mathrm{~mm}$ is selected. Then, at least eight capacitance sensors are focused on the reference cylinder with axial symmetry and at two levels (four capacitance sensors for each level). These sensors give indication concerning the lateral offset and the tilt of the reference cylinder as well as a correction in real time. Since the capacitance sensor is a surface sensor, it ensures good filtering of the possible errors on the cylindrical reference's surface (roughness and local manufacturing defects of the reference cylinder), that is why an aluminum cylinder manufactured by a diamond tool can be accepted. The acquisition of the dataset given by all probes of the machine (eight capacitance sensors, four measuring probes (to measure the part), two encoders and three optical rulers) will be synchronized. The average running of the information given by the capacitance sensors and all the other probes of the machine has to be completed similarly over a short time period of $1 \mathrm{~s}$ in order to reduce the noise and to ensure nonlinear residuals (difference between the dataset and the polynomial model) less than $2 \mathrm{~nm}$.

\subsection{Selecting measuring probes (probes focused on the artifact)}

There are many fewer options for selecting measuring probes for the cylindrical artifact than for selecting reference probes as

- the diameter of the parts may be smaller (a few millimeters), giving rise to the risk of critical offset from the target axis,

- the measurement of small holes limits the space available for the probes.

The use of combined capacitive sensors could have been considered, but any other currently available technology of probes, with contact or not, can be used. That is why measuring probes were implemented on an independent structure easily relatable to the metrological structure and that may feature an external setting station.

\subsection{Information redundancy}

The pair of reference probes (capacitance sensors), shown in figure 10, were doubled to enhance the measurements and optimize working time. The number of possible associations is 


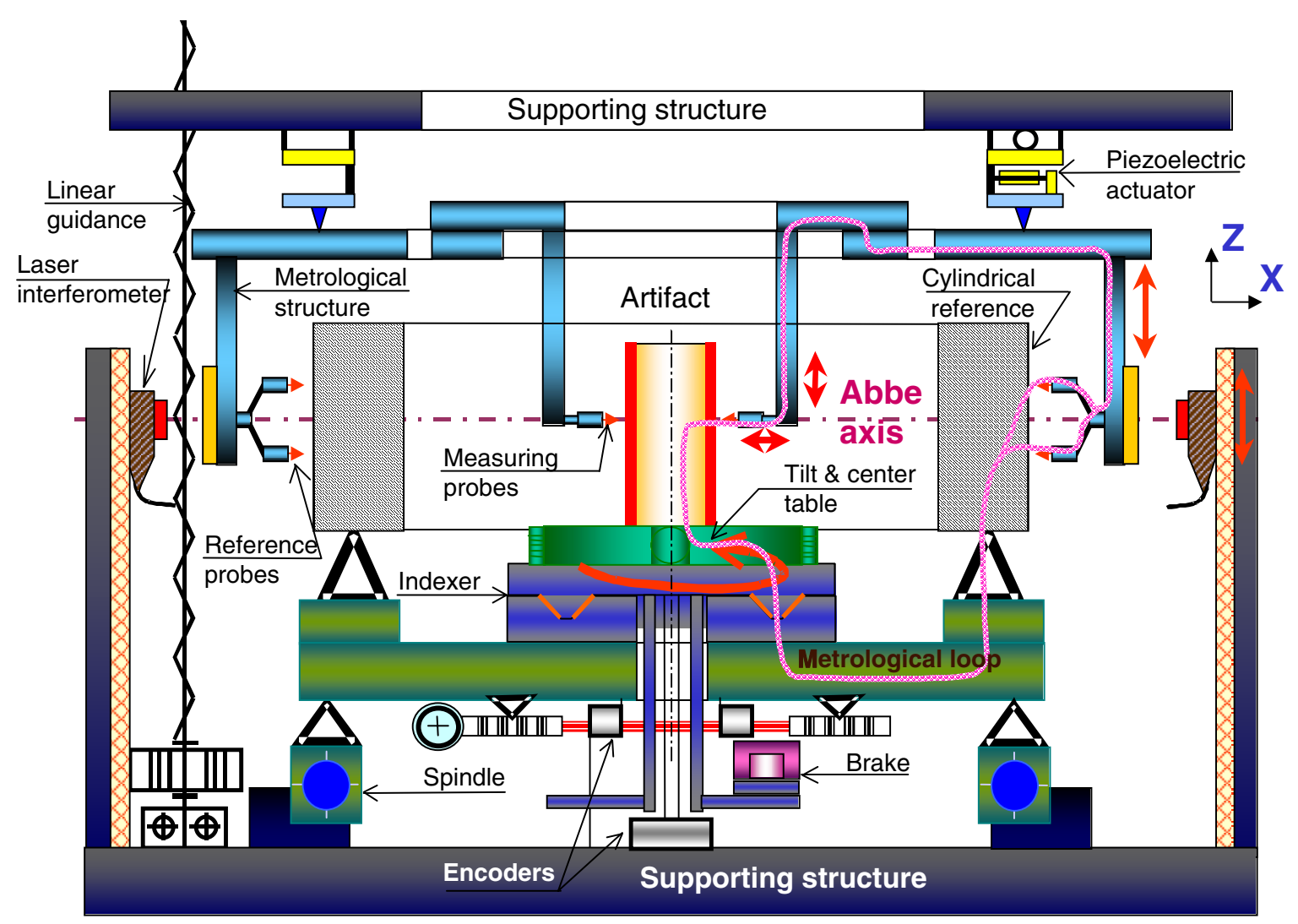

Figure 11. Architecture of the high-accuracy geometry measurement machine.

increased, and the measurement uncertainties can be evaluated accurately. Another improvement consists in mounting a second set of probes in a perpendicular plane. This amounts to creating a second independent machine for the same artifact, which further improves the measurement uncertainties.

\subsection{Architecture of the new form ultra-high precision machine}

Figure 11 represents a schematic diagram that does not show all the elements of the machine. The main revolute joint is made with a high-dimension rolling bearing. The table is linked to the rotating part of the bearing with a full isostatic linkage similar to what links the reference cylinder to the rotary table [17].

The rotation drive is an independent system that drives the table using a linkage made up of a double-sliding universal joint, under the control of a first optical encoder. In this way, the table carrying the part, which is a component of the metrology loop, is not subjected to any stress caused by the rotation.

An angular indexing system to be installed on the table allows automatic change of the angular position of the aluminum cylindrical reference in relation to the measured artifact (modified multi-step form error separation method), under the control of a second optical encoder. This angular indexing component also preserves the position of the artifact's axis with respect to the axes of both the rotary table and the aluminum reference cylinder. Special requirements are that a temperature difference between the higher part and the lower part does not affect the coaxiality of the artifact.
The tilt and center component is installed on the upper part of the angular indexing table. It is used to align the axis of the artifact and the revolute axis within a few micrometers and with perfect nanometric stability. The tilt and center component has automated settings, but no motor. The table carrying probes can perform a vertical translation movement made possible by three mechanical linear guiding systems that all have a digital command and under the control of three optical rulers.

A compound table with flexible blades, ensuring two degrees of freedom of translation along the $X$ and $Y$ axes, is used to in situ calibrate the capacitance sensors and the measuring probes. This structure is also defined to check the lateral position of the reference and measuring probes according to their respective generators as well as the mechanical centering and compensation of this error in real time. The compound table is implemented under the upper supporting structure.

Probes are fixed on the metrological structure that is located under the compound table. An isostatic linkage is used to ensure that the structure carrying probes is not subjected to deformations. The measuring probes are installed on a structure that is easy to implement and that can be set up with an external setting station. The support of the reference probes (metrological structure) is equipped with four plane mirrors, on which four laser interferometers are aligned and implemented on a separate non-deformable structure. 


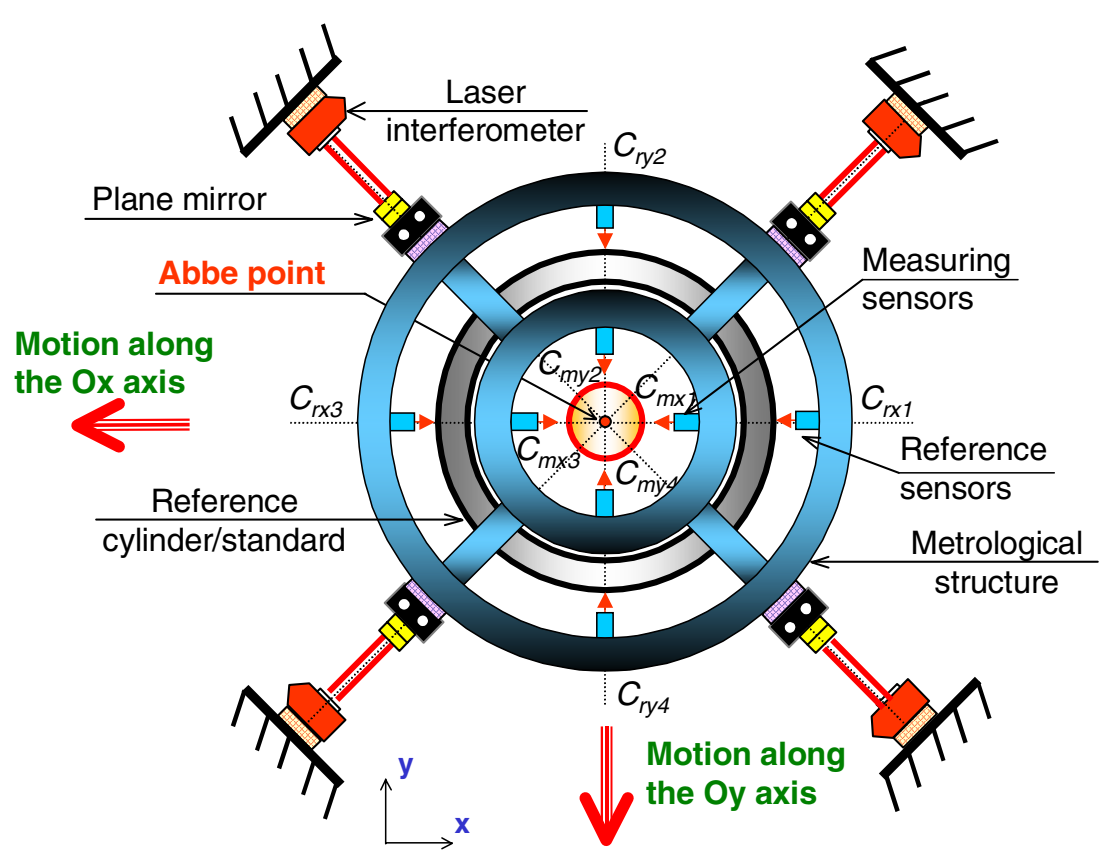

Figure 12. Schematic of the calibration structure.

\subsection{In situ calibration of the capacitance sensors and the measuring probes}

As many probes (at least eight capacitance sensors and four measuring probes) are used on cylindrical surfaces, the in situ calibration process of all the probes of the machine is planned against laser interferometers that are only used for this function of in situ calibration. Then, four laser interferometers have to be used as shown in figure 12, in order to minimize the effect of the thermal deviations.

In the general architecture of the machine, the support of the capacitance sensors and measuring probes (metrological structure) is mounted on a compound table with flexible blades. The motion is generated by two piezoelectric actuators, which can ensure nanometric motion steps over the total travel range of $100 \mu \mathrm{m}$. However, the architecture of the in situ calibration structure of the capacitive sensors and measuring probes has been studied in details by Vissiere et al [18]. An angular position offset of $45^{\circ}$ between the reference and measuring probes and the interferometers is respected. This configuration allows the uncertainty to be decreased, because each probe dataset is compared to the information given by four laser interferometers. The function of in situ calibration of the capacitance sensors and the measuring probes can be completed in a short time duration of $2 \mathrm{~min}$.

Since the distance between the laser interferometers and the mirror is approximately equal to $20 \mathrm{~mm}$, which can be helpful for the alignment of the laser beam, a mechanical structure is emphasized to protect the laser beam against external disturbance. The air disturbance can be caused mainly by the air-conditioner system, which controls both temperature and humidity around the machine, respectively, to $20 \pm$ $0.2{ }^{\circ} \mathrm{C}$ and $50 \%$. However, a compensation of both the refractive index of the air (according to the information given by a Vaisela instrument: temperature, hygrometry and pressure) and the dead path has to be considered [19, 20].
As mentioned before, the calibration of the capacitance sensors and the measuring probes as well as the nonlinear residual (which is the difference between the experiment and the polynomial model) should be completed with a nanometric level of uncertainty, less than $2 \mathrm{~nm}$. This gives rise to the need for the information given by the four laser interferometers to be stable to a nanometer. The structure joining the laser interferometers should also be stable at the nanometer level, which justifies the choice of a structure made of invar and decoupled from the supporting structure.

\section{Conclusion}

In this paper, the conventional form measurement machine for cylindrical targets is analyzed and detailed in order to determine their precision limitation, which mainly depends on the motion error, the structure deformation and the thermal drift.

The concept of dissociated metrological structure (DMT) was introduced and applied to a conventional architecture. The application and the optimization of the DMT principle by using the original concept of two-dimensional reference led to the presentation of a new architecture for an ultra-high accuracy machine and a method to calibrate it.

\section{References}

[1] David J M 1989 Machine à mesurer par coordonnées Renault Automation Patent FR 2627582-19890825)

[2] David J M and Coorevits T 1993 Les limites de la correction par logiciel des erreurs de géométrie des M.M.T. définition d'une architecture nouvelle de machine Proc. 6th Int. Conf. on Metrology

[3] Leleu S 2000 Contribution à l'évaluation des angles, conception, réalisation et validation d'un plateau pivotant de très haute précision $P h D$ Thesis Arts et Métiers Paristech, France 
[4] Lahousse L et al 2005 Application d'une nouvelle conception d'architecture à une machine de mesure de résolution nanométrique Rev. Fr. Metrol. 4 35-43

[5] Ducourtieux S and Poyet B 2011 Development of a metrological atomic force microscope with minimized Abbe error and differential interferometer-based real-time position control Meas. Sci. Technol. 22094010

[6] http://kcdb.bipm.org/AppendixC/search.asp?branch= L/DimMet

[7] Lüdicke F et al 2000 Form and length measurement by use of a modified commercial form measurement instrument American Society of Precision Engineering Annu. Meeting

[8] Abbe E 1890 Messapparte für physiker Z. Instrum. 10 446-8

[9] Neugebauer M et al 1997 A new comparator for measurement of diameter and form of cylinders, spheres and cubes under clean-room conditions Meas. Sci. Technol. 8 849-56

[10] Nouira $\mathrm{H}$ et al 2010 Investigation of the influence of the main error sources on the capacitive displacement measurements with cylindrical artefacts J. Prec. Eng. submitted

[11] Coorevits T and David J M 1991 Elimination of geometrical errors by permutations, application to a rotary table CIRP Ann. 40 531-4

[12] Marsh E R 2008 Handbook Precision Spindle Metrology (Lancaster, PA: DEStech)

[13] Nouira $\mathrm{H}$ et al 2011 Cylindrical measurement with nanometric level of accuracy: numerical comparison between the standard and the modified multi-step error separation methods Proc. Int. Conf. MacroScale: Recent Developments in Traceable Dimensional Measurements

[14] Vissiere A et al 2012 A newly conceived cylinder measuring machine and methods that eliminate the spindle errors Meas. Sci. Technol. 23094014

[15] Nouira H et al 2011 Evaluation of the capacitive displacement measurements in mechanical metrology with cylindrical artifacts Proc. 13th Int. Conf. on Metrology and Properties of Engineering Surfaces pp 300-4

[16] Vissiere A et al 2011 Evaluation of the capacitive displacement measurements in mechanical metrology with cylindrical artifacts Proc. 11th Int. Conf. EUSPEN pp 143-6

[17] David J M, Nouira H and Vailleau G P 2010 Dispositif et procédé de mesure de caractéristiques géométriques Laboratoire National de Métrologie et d'Essais (LNE) Patent FR 1057394

[18] Vissiere A et al 2011 Experimental evaluation of the dissociated metrological technique (DMT) for the geometrical measurement in metrological applications Proc. 13th Int. Conf. on Metrology and Properties of Engineering Surfaces pp 394-8

[19] Stone J, Phillips S D and Mandolfo G A 1996 Corrections for wavelength variations in precision interferometric displacement measurements J. Res. Natl Inst. Stand. Technol. 101671

[20] Jeager G 2010 Limitations of precision length measurements based on interferometers Measurement 43 652-8 